\title{
Homocysteine as an Independent Risk Factor for Cerebral Ischemic Stroke in South Indian Population in Rural Tertiary Care Centre
}

\author{
Dr. Venkata Madhav $\mathrm{M}^{1}{ }^{1}$ Dr. Anjaneya Prasad $\mathrm{V}^{2}$ Pradeep Babu KV ${ }^{3}$ \\ ${ }^{I}$ Associate Professor, Department of Internal Medicine, Dr.PSIMS\&RF, Chinoutpally, Gannavaram Mandal, \\ Krishna District, Andhra Pradesh. INDIA. 521286 \\ ${ }^{2}$ Associate Professor, Department of Internal Medicine, Dr.PSIMS\&RF, Chinoutpally, Gannavaram Mandal, \\ Krishna District, Andhra Pradesh. INDIA. 521286 \\ ${ }^{3}$ Department of Internal Medicine, Dr.PSIMS\&RF, Chinoutpally, Gannavaram Mandal, Krishna District,
} Andhra Pradesh. INDIA. 521286

\begin{abstract}
Objective:_Cerebral ischemic stroke_is a life threatening neurological disorder leads to mortality and long term disability in survival patients. Atherosclerosis is a common cause of ischemic strokes_which inturn predisposes thromboembolism and than triggers thrombotic strokes or transient ischemic attacks. The atherogenic nature of homocysteine plays a vital role in the development of cardiac or cerebral ischemic strokes. The purpose of this case - control study was to corelate homocysteine in cerebral ischemic stroke patients.
\end{abstract}

Method: This study was done at the Department of Medicine, DR. PSIMS \& RF foundation Chinaoutpally, A.P.India between March 2011 to March 2013. In this study, we recruited patients of ischemic non cardioembolic stroke with history of sudden onset of focal neurological deficit lasted more than 24 hours.

Results: The mean homocystein levels in cases were $17.58 \pm 10.3$ statistically significant than controls $6.34 \pm$ $4.22(p<0.05)$. Mean homocystein levels of cerebral ischemic stroke males were $19.21 \pm 12.1$ statistically significant than controls $7.428 \pm 4.091$ ( $p$ 0.05). Mean homocystein levels of cerebral ischemic stroke females were $14.86 \pm 5.34$ statistically significant than controls $4.536 \pm 3.844 \quad(<0.05)$. Presence of hyperhomocysteinemia in 59cases and 6 controls, statistically significant than controls $(p<0.05)$, among in males, hyperhomocysteinemia in 40 cerebral ischemic stroke patients than control (4) group, statistically significant than controls ( $p<0.05$ )

Conclusions:-Elevated serum homocysteine is a strong and modifiable risk factor of cerebral ischemic strokes. Many studies are showed the significance of development of complications like cardiac and cerebral vascular events. We support the consideration of serum homocysteine as a regular and routine screening marker to protect target organ damage.

Key_words: stroke, homocysteine, ischemia, thrombosis

\section{Introduction:}

A cerebrovascular stroke has become a major health problem and is the most common cause of mortality and morbidity in the entire world. Stroke is defined as sudden occurrence of clinical symptoms and or signs of either focal or global loss of cerebral function with the duration of more than 24 hours or leading to mortality with no other reason than vascular origin ${ }^{1 .}$ The global burden of disease (GBD) study, in 1990, reported 9.4 million deaths in India of which 61900 were from stroke and disability adjusted life years (DALYs) lost to 28.5 million. Recent epidemiological surveys for cerebrovascular diseases like hemiplegia or paraplegia, showed 320 cases in 145456 persons indicating an overall crude prevalence rate of 220 per 100000 persons ${ }^{2}$.The broad classification of strokes, primarily in to ischemic $(85 \%)$ and hemorrhagic $(15 \%){ }^{3}$, of ischemic strokes commonest etiologies include atherosclerosis with thromboembolism and cardiogenic thromboembolism. The modifiable personal or social risk factors like hypertension, diabetes, obesity and cigarette smoking triggers proportional incidence of cerebrovascular events, like stroke and TIA ${ }^{3}$. The modifiable biochemical risk factors like abnormal lipid metabolism ${ }^{4}$, hypercholesterolemia, lipoprotein (a) $[\mathrm{Lp}(\mathrm{a})]^{5}$, hyperhomocysteinemia ${ }^{6}$ for increased incidence of atherothrombotic cerebrovascular disease. Hyperhomocysteinemia recently has been recognised as a easily modifiable risk factor for the presence of atherosclerotic cerebro or cardiovascular disease and hypercoagulability states. ${ }^{7}$ In humans, less severe genetic mutations associated with enzyme abnormalities particularly methylenetetrahydrofolate reductase $(M T H F R)$ in the metabolic pathways involving folate and homocysteine leads to elevated levels of homocysteine ${ }^{8}$. Elevated homocysteine levels play a causal role in the pathogenesis of atherosclerosis, thrombo-embolism and vascular endothelial dysfunction ${ }^{9}$ with an increased incidence of ischaemic stroke. An other positive correlation for the observed association between 
hyperhomocysteinemia and stroke is a additive effect of factors associated with hyperhomocysteinaemia particularly with cigarette smoking, renal dysfunction, an atherogenic diet, cystine or folate deficiency triggers the acute vascular events themselves, whereby the vascular endothelial damage increases total homocysteine levels ${ }^{9}$. Other mechanisms, by which total homocysteine induces vascular changes include high propensity for development of thrombus, impaired thrombolysis, increased production of hydrogen peroxide, and increased oxidation of LDL - cholesterol are responsible for development of vascular endothelial dysfunction ${ }^{10}$.

On this background, our study aims at comparing the occurrence of homocysteine levels in patients with cerebral ischemic stroke group, is to determine the role of homocysteine as a marker for ischemic stroke, with regard to Indian population.

\section{Material and methods:}

A case - control study was undertaken in the Department of General Medicine, Dr.PSIMS\&RF, Chinoutpally, Gannavaram, Krishna (Dt), Andhra Pradesh, during the period of two years i.e., March 2011 to March 2013 with the diagnosis of acute cerebral ischemic stroke as per history of sudden onset of focal neurological deficit lasted more than 24 hours. A detailed history and thorough clinical examination was done as per the proforma and were investigated further.

The levels of homocysteine were measured by Chemiluminescent microparticle immunoassay (CMIA) in acute cerebral ischemic stroke patients and age and sex matched controls. The levels were analyzed and compared between different groups.

\section{Inclusion criteria:}

Clinical evidence of stroke

Cranial computed tomography (CT) scan or MR imaging consistence with ischemic stroke

\section{Exclusion criteria:}

Patients with CT scan or MR imaging showing hemorrhage or mass lesion

Patients with Coronary artery disease

Patients with Vasculitis

Patients with other endocrine, liver and renal diseases

\section{Sample collection and analysis:}

Both heparinised and plain blood samples were collected from each case and control. For analysis of FBS, lipid profile, - serum was used. Complete haemogram, Urine Routine, ECG, Serum Homocysteine Levels, Blood urea, serum creatinine, Liver function tests, Serum glucose estimation was done by Trindler's GOD - POD method (commercial kit - ERBA - MANNHEIM), All these estimations were performed by Randox Daytona Autoanalyzer.

Estimation of serum homocysteine: Homocysteine assay is a chemiluminescent microparticle immunoassay (CMIA) for the quantitative determination of total L-homocysteine in human serum or plasma on the ARCHITECT i System optics.

According to Kang et al. an abnormal homocysteine level is defined by an arbitrary cut off (95th percentile) of the concentration found in normal population. The normal fasting plasma homocysteine levels in adults usually range between $5-15 \mu \mathrm{mol} / \mathrm{L}$ with mean level of about10 $\mu \mathrm{mol} / \mathrm{L}^{11}$, Kang and co-workers have classified hyperhomocystenemia as $>15 \mu \mathrm{mol} / \mathrm{L}$.

\section{Results:}

The mean age in cases and controls was $56.26 \pm 12.57$, among in males was $54.4 \pm 12.56$ and in females was $59.36 \pm 12.06$, the percentage of males $(62.4 \%)$ were higher than females $(37.6 \%)$. The mean BMI in cases was $23.8 \pm 1.01$ and in controls was $23.6 \pm 1.19$. Presence of hyperhomocysteinemia in $59(41.8 \%)$ of cerebral ischemic stroke patients than control group $6(4.25 \%)$, among in male cerebral ischemic stroke patients $40(45.4 \%)$ than control group 4 (4.54\%), among in female cerebral ischemic stroke patients $19(35.8 \%)$ than control group $2(3.77 \%)$ were statistically significant. The mean homocystein levels in cases were $17.58 \pm 10.3$ statistically significant than controls $6.34 \pm 4.22(\mathrm{p}<0.05)$. Mean homocystein levels of cerebral ischemic stroke males were $19.21 \pm 12.1$ statistically significant than controls $7.428 \pm 4.091$ (p 0.05). Mean homocystein levels of cerebral ischemic stroke females were $14.86 \pm 5.34$ statistically significant than controls $4.536 \pm 3.844$ $(<0.05)$. Presence of hyperhomocysteinemia in 59cases and 6 controls, statistically significant than controls $(\mathrm{p}$ $<0.05$ ), among in males, hyperhomocysteinemia in 40 cerebral ischemic stroke patients than control (4) group, statistically significant than controls ( $\mathrm{p}<0.05)$. Among in females, hyperhomocysteinemia in 19 cerebral ischemic stroke patients than control (2) group, statistically significant than controls $(\mathrm{p}<0.05)$. The risk factors 
are present in cerebral ischemic stroke patients, hypertension 31(21.9\%), diabetes $26(18.4 \%)$, dyslipidemia 22 $(15.6 \%)$, smoking $14(15.9 \%)$ and no risk factors in $48(34.04 \%)$, among the risk factors and non risk factors group, the incidence of hyperhomocysteinemia in hypertension $12(38.7 \%)$, diabetes $8(30.7 \%)$, dyslipidemia 7 $(31.8 \%)$, smoking $5(35.7 \%)$ and no risk factors in $27(56.25 \%)$ were statistically significant. The mean CRP levels in cases were $3.74 \pm 2.44$ statistically significant than controls $2.09 \pm 0.905$ (p: 0.05). Mean CRP levels of cerebral ischemic stroke males were $4.13 \pm 2.46$ statistically significant than controls $2.06 \pm 0.89$ (p 0.05). Mean CRP levels of cerebral ischemic stroke females were $3.11 \pm 2.28$ statistically significant than controls $2.13 \pm 0.93$ $(<0.001)$. The mean cholesterol in cases was $196.46 \pm 32.72$ and in controls was $177.27 \pm 21.82(\mathrm{p}<0.05)$ which showed cholesterol is more significant elevation in cerebral ischemic stroke patients, The mean triglyceride level in cases was $195.54 \pm 76.78$ and in controls was $127.61 \pm 56.52(\mathrm{p}<0.05)$ which showed mean triglyceride level is more significant elevation in cerebral ischemic stroke patients, The mean LDL - cholesterol level in cases was $146.23 \pm 37.8$ and in controls was $104.57 \pm 21.52(\mathrm{p}<0.05)$ which showed mean LDL - cholesterol level is more significant elevation in cerebral ischemic stroke patients, The mean HDL - cholesterol level in cases was $33.12 \pm 7.36$ and in controls was $37.65 \pm 8.38$ ( $\mathrm{p}<0.05)$ which showed mean HDL - cholesterol level is more significant reduction in cerebral ischemic stroke patients.

Table 1

Presence of Hyperhomocysteinemia in cases and controls

\begin{tabular}{|l|l|l|l|l|}
\hline & Cases & Controls & $\begin{array}{l}\text { Chi square } \\
\text { value }\end{array}$ & P value \\
\hline Total & 141 & 141 & 54.061 & $<0.0001$ \\
\cline { 1 - 3 } Hyperhomocystenemia & 59 & 6 & & \\
\hline
\end{tabular}

Chi squared equals 54.061 with 1 degrees of freedom. The two-tailed $\mathrm{P}$ value is less than 0.0001 The association between rows (groups) and columns (outcomes) is considered to be extremely statistically significant.

Table 2

Mean homocystein levels in cases and controls

\begin{tabular}{|l|l|l|l|}
\hline & Cases & Controls & P value \\
\hline Male & $19.21 \pm 12.1$ & $7.428 \pm 4.091$ & $<0.05$ \\
\hline Female & $14.86 \pm 5.34$ & $4.536 \pm 3.844$ & $<0.05$ \\
\hline Total mean & $17.58 \pm 10.3$ & $6.34 \pm 4.22$ & $<0.05$ \\
\hline
\end{tabular}

Table 3

Presence of Hyperhomocysteinemia in cases and controls in males

\begin{tabular}{|l|l|l|l|l|}
\hline & Cases & Controls & $\begin{array}{l}\text { Chi square } \\
\text { value }\end{array}$ & P value \\
\hline Total & 88 & 88 & 37.121 & $<0.0001$ \\
\hline Males & 40 & 4 & & \\
\hline
\end{tabular}

Chi squared equals 37.121 with 1 degrees offreedom. The two-tailed $\mathrm{P}$ value is less than $0.0001 \overline{\text { The association }}$ between rows (groups) and columns (outcomes) is considered to be extremely statistically significant

Table 4

Presence of Hyperhomocysteinemia in cases and controls in females

\begin{tabular}{|l|l|l|l|l|}
\hline & Cases & Controls & $\begin{array}{l}\text { Chi square } \\
\text { value }\end{array}$ & P value \\
\hline Total & 53 & 53 & 15.202 & $<0.0001$ \\
\hline Females & 19 & 2 & & \\
\hline
\end{tabular}

Chi squared equals 15.202 with 1 degrees of freedom. The two-tailed P value is less than $0.0001 \overline{T h e}$ association between rows (groups) and columns (outcomes) is considered to be extremely statistically significant. 
Table 5

Risk factors in cases

\begin{tabular}{|l|l|l|}
\hline \multicolumn{1}{|c|}{ Risk factors } & No of cases & Percentage $(\%)$ \\
\hline Hypertension & 31 & $21.9 \%$ \\
\hline Diabetes & 26 & $18.4 \%$ \\
\hline Dyslipidemia & 22 & $15.6 \%$ \\
\hline Smoking & 14 & $15.9 \%$ \\
\hline No risk factors & 48 & $34.04 \%$ \\
\hline
\end{tabular}

Table 6

Presence of Hyperhomocystenemia in cases with or without risk factors

\begin{tabular}{|l|l|l|l|l|}
\hline Risk factors & No of cases & $\begin{array}{c}\text { Hyperhomocysteinemia } \\
(\%)\end{array}$ & $\begin{array}{l}\text { Males } \\
\text { Hcy }\end{array}$ & $\begin{array}{l}\text { Females } \\
\text { Hcy }\end{array}$ \\
\hline Hypertension & 31 & $12(38.7 \%)$ & 7 & 5 \\
\hline Diabetes & 26 & $8(30.7 \%)$ & 5 & 3 \\
\hline Dyslipidemia & 22 & $7(31.8 \%)$ & 3 & 4 \\
\hline Smoking & 14 & $5(35.7 \%)$ & 5 & 0 \\
\hline No risk factors & 48 & $27(56.25 \%)$ & 20 & 7 \\
\hline Total & 141 & $59(41.8 \%)$ & 40 & 19 \\
\hline
\end{tabular}

Table 7

Presence of Hyperhomocysteinemia in cases with or without risk factors

\begin{tabular}{|l|l|l|l|l|}
\hline & $\begin{array}{l}\text { With risk } \\
\text { factors }\end{array}$ & $\begin{array}{l}\text { without risk } \\
\text { factors }\end{array}$ & $\begin{array}{l}\text { Chi square } \\
\text { value }\end{array}$ & Palue \\
\hline Total & 93 & 48 & 5.341 & $<0.0208$ \\
\hline Hyperhomocystenemia & $32(34.4 \%)$ & $27(56.25 \%)$ & & \\
\hline
\end{tabular}

Chi squared equals 5.341 with 1 degrees of freedom. The two-tailed $\mathrm{P}$ value equals 0.0208 The association between rows (groups) and columns (outcomes) is considered to be statistically significant.

Table 8

Mean CRP levels in cases and controls

\begin{tabular}{|l|l|l|l|}
\hline & Cases & Controls & P value \\
\hline Male & $4.13 \pm 2.46$ & $2.06 \pm 0.89$ & $<0.05$ \\
\hline Female & $3.11 \pm 2.28$ & $2.13 \pm 0.93$ & $<0.05$ \\
\hline Total mean & $3.74 \pm 2.44$ & $2.09 \pm 0.905$ & $<0.05$ \\
\hline
\end{tabular}

\section{Discussion:}

In this study, serum hyperhomocystenemia was associated with an increased risk of cerebral ischemic strokes, patients without associated risk factors. Although many studies have previously shown the association between hyperhomocystenemia and stroke patients with associated risk factors. The present study was focused on patients without associated risk factors (34.04\%), had developed hyperhomocystenemia in $56.25 \%$ of patients. The mean age in cerebral ischemic stroke patients was $56.26 \pm 12.57$, and $71.7 \%$ cerebral ischemic stroke patients were noticed more between the age group of $41-60$ years. This study shows the mean serum homocystene was more in cerebral ischemic stroke group $17.58 \pm 10.3$ than compared to normal subjects $6.34 \pm$ 4.22, when compared with L. Parnetti et al study, ${ }^{12}$ the results were similar that is more in patients with large vessel disease group. Presence of hyperhomocysteinemia in $59(41.8 \%)$ of cerebral ischemic stroke patients than control group $6(4.25 \%)$, supported by Carod-Artal FJ et al ${ }^{13}$ study. Males showed increase in mean serum homocystene levels and increased incidence compared to females in cerebral ischemic stroke group, supported by Carod-Artal FJet al $^{13}$ study. The risk factors like hypertension, diabetes, dyslipidemia and smoking are association of incidence of hyperhomocystenemia in $34.4 \%$ of cerebral ischemic stroke patients, the risk increases significantly if associated with smoking in males ${ }^{13}$. Mean CRP levels were more in cerebral ischemic stroke patients $3.74 \pm 2.44$ compared to controls $2.09 \pm 0.905$. CRP levels were found to be significant elevation of $54.2 \%$ in cerebral ischemic stroke patients with hyperhomocystenemia than elevated CRP levels were found in $35.4 \%$ of cerebral ischemic stroke patients without hyperhomocystenemia ${ }^{14}$. Males showed increase in mean 
serum CRP levels and homocystene levels compared to females in cerebral ischemic stroke patients. Serum total cholesterol, triglycerides and LDL were elevated in cerebral ischemic stroke patients compared to controls and HDL levels were decreased in cerebral ischemic stroke patients group compared to controls ${ }^{15}$. Among 141 patients with cerebral ischemic stroke 59 patients $(41.8 \%)$ have hyperhomocystenemia and 50 patients $(35.4 \%)$ showed high CRP levels, out of which in 59 patients with hyperhomocystenemia in cerebral ischemic stroke patients 32 had high CRP levels with a p value of 0.0002.Among 141 controls , 6 people(4.25\%) have hyperhomocystenemia and out of which in 3 showed high CRP levels with a p value of $<0.05^{16}$.

\section{Conclusions:}

Homocystene levels were found to be significantly higher in cerebral ischemic stroke patients by 41.8\%. $71.7 \%$ of cerebral ischemic stroke patients belong to $41-60$ years age group. Male to female ratio were found to be 1.66: 1.high incidence of hyperhomocystenemia in $56.25 \%$ of cerebral ischemic stroke patients without risk factors than patients with hypertension, diabetes, dyslipidemia and smoking associated incidence of hyperhomocystenemia in $34.4 \%$ of cerebral ischemic stroke patients. Elevated serum homocysteine is a strong and modifiable risk factor of cerebral ischemic strokes. Many studies are showed the significance of development of complications like cardiac and cerebral vascular events. We support the consideration of serum homocysteine as a regular and routine screening marker to protect target organ damage.

\section{Bibliography:}

[1] Hatano S. Experience from a multicentre stroke register: a preliminary report. Bull World Health Organ 1976; 54:541-553.

[2] Dalal PM. Strokes in young and elderly: risk factors and strategies for stroke prevention. J Assoc Phys India 1997;. 45:125-31.

[3] Itrat A, Ahmed B, Khan M, Muhammad M, Risk factor profiles of South Asians with cerebrovascular disease Int J Stroke. 2011 Aug;6(4):346-8.10.1111/j.1747-4949.2011.00622.x

[4] Qian Jia, MD, Liping Liu, MD, PhD, Yongjun Wang, Risk Factors and Prevention of Stroke in the Chinese Population: Journal of Stroke and Cerebrovascular Diseases Volume 20, Issue 5, September-October 2011, Pages 395-400

[5] Baidarbhi Chakraborty, Gaurav Vishnoi, Binita Goswami, Lipoprotein(a), Ferritin, and Albumin in Acute Phase Reaction Predicts Severity and Mortality of Acute Ischemic Stroke in North Indian Patients: Journal of Stroke and Cerebrovascular Diseases 2012.10.013,10.1016

[6] Bertsch T, Mielke O, Höly S, Zimmer W, Casarin W, Homocysteine in cerebrovascular disease: an independent risk factor for subcortical vascular encephalopathy:Clin Chem Lab Med. 2001 Aug;39(8):721- 4

[7] Abraham R, John MJ, Calton R, Dhanoa J. Raised serum homocysteine levels in patients of coronary artery disease and the effect of vitamin b12 and folate on its concentration. Indian Journal Of Clinical Biochemistry 2006;21(1):95-100.

[8] Elizabeth A. Varga, MS; Amy C. Sturm, MS; Caron P. Misita, PharmD;Stephan Moll, MD Homocysteine and MTHFR Mutations Relation to Thrombosis and Coronary Artery Disease Circulation. 2005; 111: e289-e293

[9] Hanke, Graeme J.; Eikelboom, John W: Homocysteine and stroke: Current Opinion in Neurology:February 2001 - Volume 14 Issue 1 - pp 95-102

[10] Chambers JC, McGregor A, Jean-Marie J, Obeid OA, Kooner JS. Demonstration of rapid onset vascular endothelial dysfunction after hyperhomocysteinemia: an effect reversible with vitamin C therapy. Circulation. 1999;99:1156-1160

[11] Kang SS, Wong PWK, Malinow MR. Hyperhomocysteine as a risk factor for occlusive vascular disease. Ann Rev Nut 1992; 12:279-298.

[12] L. Parnetti, V.Caso, ,A.Santucci, F. Corea, A. Lanari, A. Floridi, Mild hyperhomocysteinemia is a risk-factor in all etiological subtypes of stroke Neurological Sciences April 2004, Volume 25, Issue 1, pp 13-17

[13] Carod-Artal FJ, Nunes SV, Vargas AP, Portugal D. Factors determining hyperhomocysteinemia in the chronic phase of strokes: Rev Neurol. 2007 May 1-15;44(9):513-9.

[14] Larry B. Goldstein MD: Novel risk factors for stroke: Homocysteine, inflammation, and infection: Current Atherosclerosis Reports: 2000, Volume 2, Issue 2, pp 110-114

[15] Charles J. Glueck, MD Peter Shaw, MD, James E. Lang, MD, Trent Tracy, PA, Luann Sieve-Smith,Ying Wang, MS: Evidence that homocysteine is an independent risk factor for atherosclerosis in hyperlipidemic patients: The American Journal of CardiologyVolume 75, Issue 2, 15 January 1995, Pages 132-136

[16] Mária T. Magyar, MD; Zita Szikszai, MS; József Balla: Early-Onset Carotid Atherosclerosis Is Associated With Increased IntimaMedia Thickness and Elevated Serum Levels of Inflammatory Markers: Stroke: 2003; 34: 58-63 